\title{
General Diseases Influence on Peri-Implantitis Development: a Systematic Review
}

\author{
Zygimantas Guobis $^{1}$, Ingrida Pacauskiene ${ }^{1}$, Inesa Astramskaite ${ }^{2}$ \\ ${ }^{1}$ Department of Oral and Dental Pathology, Lithuanian University of Health Sciences, Kaunas, Lithuania. \\ ${ }^{2}$ Department of Maxillofacial Surgery, Lithuanian University of Health Sciences, Kaunas, Lithuania.
}

\author{
Corresponding Author: \\ Zygimantas Guobis \\ Laisves al. 92a-2, LT- 44251, Kaunas \\ Lithuania \\ Phone: 0037061385620 \\ E-mail: zygimantasg@yahoo.com
}

\begin{abstract}
Objectives: To systematically review the influence of systemic diseases or medications used in their treatment on the dental implant therapy success.

Material and Methods: The search strategy was implemented on the National Library of Medicine database (MEDLINE) (Ovid) and EMBASE electronic databases between January 2006 and January 2016. Human studies with available English articles analysing the relationship between dental implant therapy success and systemic diseases, such as diabetes mellitus, AIDS/HIV, rheumatoid arthritis, osteoporosis, Crohn's disease, cardiovascular diseases, scleroderma, Sjögren's syndrome, lichen planus, ectodermal dysplasia, post-transplantation status, were included in present review according to the PRISMA guidelines. The review protocol was registered on PROSPERO system with the code CRD42016033662.

Results: Present review included forty one retrospective and prospective follow-up studies, case-control studies, case report series and cohort studies. Despite some limitations this study reveals positive results of implantation in most systemic conditions that should be interpreted with caution. Influence of cardiovascular diseases on the dental implantation success should be explored deeply, because of controversial results and likelihood of comorbidity expressed by a history of cardiovascular diseases and periodontitis. There is only a weak relationship with bone density in osteoporosis and implant failure. All the other diseases did not show significant effect on implantation success.

Conclusions: Recent studies with low strength of evidence and controversy show that systemic diseases may have potential effect on the success of implantation, but further detailed studies are needed to provide these findings.
\end{abstract}

Keywords: dental implants; diabetes mellitus; osteoporosis; peri-implantitis; rheumatoid arthritis.

\author{
Accepted for publication: 22 August 2016 \\ To cite this article: \\ Guobis Z, Pacauskiene I, Astramskaite I. \\ General Diseases Influence on Peri-Implantitis Development: a Systematic Review \\ J Oral Maxillofac Res 2016;7(3):e5 \\ URL: http://www.ejomr.org/JOMR/archives/2016/3/e5/v7n3e5.pdf \\ doi: $\underline{10.5037 / j o m r .2016 .7305}$
}




\section{INTRODUCTION}

The replacement of missing teeth with endosseous implants for the rehabilitation of edentulous or partially edentulous patients has become a standard of care in the past two decades. To achieve and maintain osseointegration, indications and contraindications must be carefully balanced, and proper patient selection is a key issue in treatment planning [1].

Osseointegrated dental implants have a long-term success rate of over $90 \%$, but may be threatened by peri-implant mucositis and peri-implantitis, bacteria biofilm-induced inflammatory conditions. A number of risk indicators were identified including poor oral hygiene, a history of periodontitis, diabetes and smoking [2-4]. Peri-implant diseases share similar aetiology and risk factors as chronic periodontitis [5]. Both of them are initiated by the accumulation of microbial biofilms on hard surfaces that is teeth or dental implants. Systemic diseases may impair the host's barrier function and immune defence against periodontal pathogens creating the opportunity for destructive periodontal disease and likely periimplantitis $[\underline{6}, \underline{7}]$.

Systemic diseases and medications or other therapies used in their treatment may also interfere healing by influencing the host tissues and physiology, thus increasing the risk of early and late failures of implants $[\underline{8}, \underline{\underline{ }}]$.

Buser et al. [10] proposed to subdivide risky diseases for implant therapy success into two groups with high risk for patients with serious systemic diseases (rheumatoid arthritis, osteomalacia, osteogenesis imperfecta, immunocompromising diseases) or alcoholism, and significant risk for patients with irradiated bone, severe diabetes mellitus, bleeding disorders or heavy smoking habits.

Although clinically relevant impact of the most common systemic diseases on the success of implant therapy has been suggested, however there is limited information on the association between implant success and systemic diseases and what type of evidence these statements are based [9].

The aim of present systematic review was to assess the influence of systemic diseases or medications used for the treatment of systemic disease, on the dental implant therapy success.

\section{MATERIAL AND METHODS Protocol and registration}

The methods of the analysis, inclusion and exclusion criteria were specified in advance and documented in a protocol. The review was registered in PROSPERO, an international prospective register of systematic reviews.

The protocol can be accessed at:

http://www.crd.york.ac.uk/PROSPERO/display record.asp?ID=CRD42016033662.

Registration number: CRD42016033662.

The reporting of this systematic analysis adhered to the PRISMA (Preferred Reporting Item for Systematic Review and Meta-Analyses) Statement [11].

\section{Focus question}

The following review question was developed according to the population, intervention, comparison, and outcome (PICO) study design: What is the risk level for implant failure or peri-implantitis in patients undergoing implant treatment with a history of scleroderma, Sjögren's syndrome, neuropsychiatric disorders/Parkinson's disease, oral lichen planus, HIV infection, ectodermal dysplasia, long-term immunosuppression after organ transplantation, cardiovascular disease, Crohn disease, diabetes, osteoporosis, medication with oral bisphosphonates, or xerostomia?

\section{Information sources}

The search strategy was based on electronic database examination. A search was implemented on the National Library of Medicine database (MEDLINE) (Ovid) and EMBASE databases based on the search strategy described further.

The search strategy included 3 stages. In the first stage, a title screening took place to exclude all irrelevant publications, case reports, reviews, and animal studies. In the second stage, the abstracts were read to see if the aim of the study met the systematic review questions. The third stage was reading full-text articles to confirm each study's eligibility, according to the inclusion and exclusion criteria. The search was conducted including studies between January 2006 and January 2016 to ensure sufficient and large enough amount of included studies.

\section{Search}

The following search strategy using keywords: "oral implant" OR "dental implant" OR "peri-implantitis" AND "osteoporosis" OR "osteoporotic" OR "oral bisphosphonates" OR "bisphosphonates" OR "diabetes mellitus" OR "insulin therapy" OR "glucose intolerance" OR "rheumatoid arthritis" OR "AIDS" or 
"HIV" OR "scleroderma" OR "Sjögren's syndrome" OR "Lichen planus" OR "neuropsychiatric disorders" OR "Parkinson" OR "ectodermal dysplasia" OR "Crohn's disease" OR "transplantation" OR "cardiovascular" OR "xerostomia"

This search for papers was intended for high initial numbers rather than highly specific and strictly rejected in the first instance. The variable terming of condition in interest was chose to broaden the search field.

\section{Selection of studies}

Three independent reviewers screened titles and abstracts of the search results. Additional studies were screened by scanning the references cited in the retained papers and by personal communication. Any disagreement regarding inclusion was resolved by two against one. The agreement between reviewers was calculated using kappa $(\kappa)$ index [12]. On the title and abstracts screening independent researchers carried out the search on various diseases. Only accepted abstracts were then discussed through all three authors. At the full-text reading stage all the researchers were included in evaluation of every study.

\section{Types of publications}

The review included all human studies and case reports series published in English. Reviews, letters, editorials and $\mathrm{PhD}$ thesis were excluded.

\section{Types of studies}

Present review included all retrospective and prospective follow-up studies, case-control studies, case report series and cohort studies published between January 2006 and January 2016, evaluating the relationship between implant treatment success and at least one systemic disease.

\section{Types of participants/population}

The subjects in the included studies should have been implantation patients who were diagnosed with any systemic disease that may affect implant treatment outcomes.

\section{Disease definition}

Authors of present review selected possibly relevant diseases that may affect the success of implantation. It was decided that successful implantation should be, when implants were stable and osseointegrated without any signs of critical complications.

\section{Inclusion and exclusion criteria}

All the included studies should have followed the inclusion criteria listed below:

- Human subjects with the respective diagnosis;

- Subjects with osseointegrated dental implants or implant placement;

- Reports of implant failure, survival, and/or success or peri-implantitis;

- Case series including at least ten subjects with the respective diagnosis. If case reports with fewer treated subjects were the only available source of information, they were listed;

- Articles available in English.

Following exclusion criteria were applied to all review studies:

- Subjects not uniformed with the diagnosis;

- Uniformed implantation procedure, whether bone augmentation, sinus lift or other additional procedures are included, or implants solely;

- Smokers, bad oral hygiene, immediate loading, immediate placement.

\section{Sequential search strategy}

On the first stage of research, same filters were added to every independent disease search: human studies, articles available, 10 years. On the initial search any case reports, reviews and other irrelevant studies were excluded. Following the abstract reading stage, inclusion and exclusion criteria were applied to the information given in abstracts, is any information was missing the full-text reading was performed. At the final stage, all the included articles were carefully screened and only relevant articles were included for further analysis.

\section{Data items}

All included studies were arranged according to following fields:

- "Author/year" - to show the author and year of publication.

- "Implantation type" - described weather the immediate implantation post-extraction, sinus lift, bone augmentation etc. were applied to included patients.

- "Number of subjects" - evaluated the number of subjects included in studies.

- "Number of implants" - showed the amount of implants analysed in studies. 
- "Year of follow-up" - described the mean, minimum and maximum follow-up time periods applied to subjects.

- "Disease severity or medication dose level" was used to evaluate the disease's severity or medication dose level and type of consumption to reveal if these factors affect the success of implantation.

- "Disease manifestations according to the implantation" - showed, weather the time (post and pre-operative) of disease manifestation affected the implantation success.

\section{Risk of bias assessment}

The quality of all included studies was evaluating during the full text reading stage. To assess the risk of bias in every study the Cochrane Collaboration's two-part tool was used [13]. Studies were assessed independently by authors. If any uncertainties were found in studies, the decision was made through the discussion between all the authors.

All the articles were individually evaluated to assess the risk of bias in random sequence generation, allocation concealment, blinding of participants and practitioners, blinding of outcomes assessment, incomplete outcomes data, selective reporting and other sources of bias. All the categories were rated as "low risk of bias", if any uncertainties were found, "high risk of bias" if study showed bias in selected category and "unclear risk of bias" if the methodology was not clearly stated and the risk remained questionable. A study itself was mentioned as "low risk of bias", if all the categories were rated for "low risk of bias". If at least one category was rated as "high risk of bias" or "unclear risk of bias", the study itself is rated as "high risk of bias" or "unclear risk of bias" respectively.

All the retrospective studies were rated with appreciation that not all the factors, that may affect the bias, could have been controlled by authors, since information of previously performed procedures was collected.

\section{Statistical analysis}

A meta-analysis integrates the quantitative findings from separate but similar studies, and it allows evaluation of the numerical results of the overall effect of interest. All meta-analysis were performed on studies reporting comparable outcomes. However, most studies could not be compared due to methodological heterogeneity and presented results dispersion. The data was analysed with a Student t-test, 95\% confidence interval (CI) was calculated where possible. To evaluate the degree of agreement between authors, $\kappa$ index was used [12].

\section{RESULTS \\ Study selection}

Study selection process was graphically designed according to PRISMA flow diagram. (Figure 1) The initial search identified a total of 1410. One additional article was added, resulting in 1401 studies in primary search stage. The screening of article titles and independent screening of abstracts resulted in 721 possibly included articles. The inclusion and exclusion criteria were applied to 82 full-text articles. A total of 41 articles that met the inclusion criteria were selected for systematic review [14-54].

The $\kappa$ values for inter-reviewer agreement for potentially relevant articles were 1 (titles and abstracts) and $>0.9$ (full-text articles), indicating an "almost perfect agreement" between the 2 reviewers [12].

\section{Study exclusion}

At the full text reading stage, in total 41 articles were excluded. The reasons of exclusion were as follows: review papers $(n=8)$, single case reports $(n=18)$, methodological and design faults $(n=15)$. Mostly exclusion should be provided due to the inadequate articles filtering at the first study selection stage.

\section{Study characteristics}

In total 16 prospective studies $[14,18,19,28,35-$

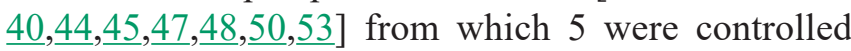
clinical trials $[\underline{38-40}, \underline{44}, \underline{48}]$ and 1 cross-sectional study [45], 23 retrospective studies $[\underline{15-17}, \underline{20}, \underline{23-}$ 27, 29-34, $41-43,46, \underline{49}, \underline{51}, \underline{52}, \underline{54]}$ and two case series studies $[21,22]$ were included in present research.

In total 6813 patients and 19332 implants were screened in included studies. However, in few articles number of implants was not listed [18, 27, $\underline{47}, \underline{51-53}]$. The follow-up mean period varied from 9 weeks up to 10 years. However, in few studies follow-up period was not listed due to methodological differences $[23,24,26,29,30,32]$.

The implantation success ratio ranged between $100 \%$ and $73 \%$ on patients and between $100 \%$ and $82.6 \%$ on implants. However, these results should be interpreted carefully, since very wide follow-up period gap was seen in included studies. 


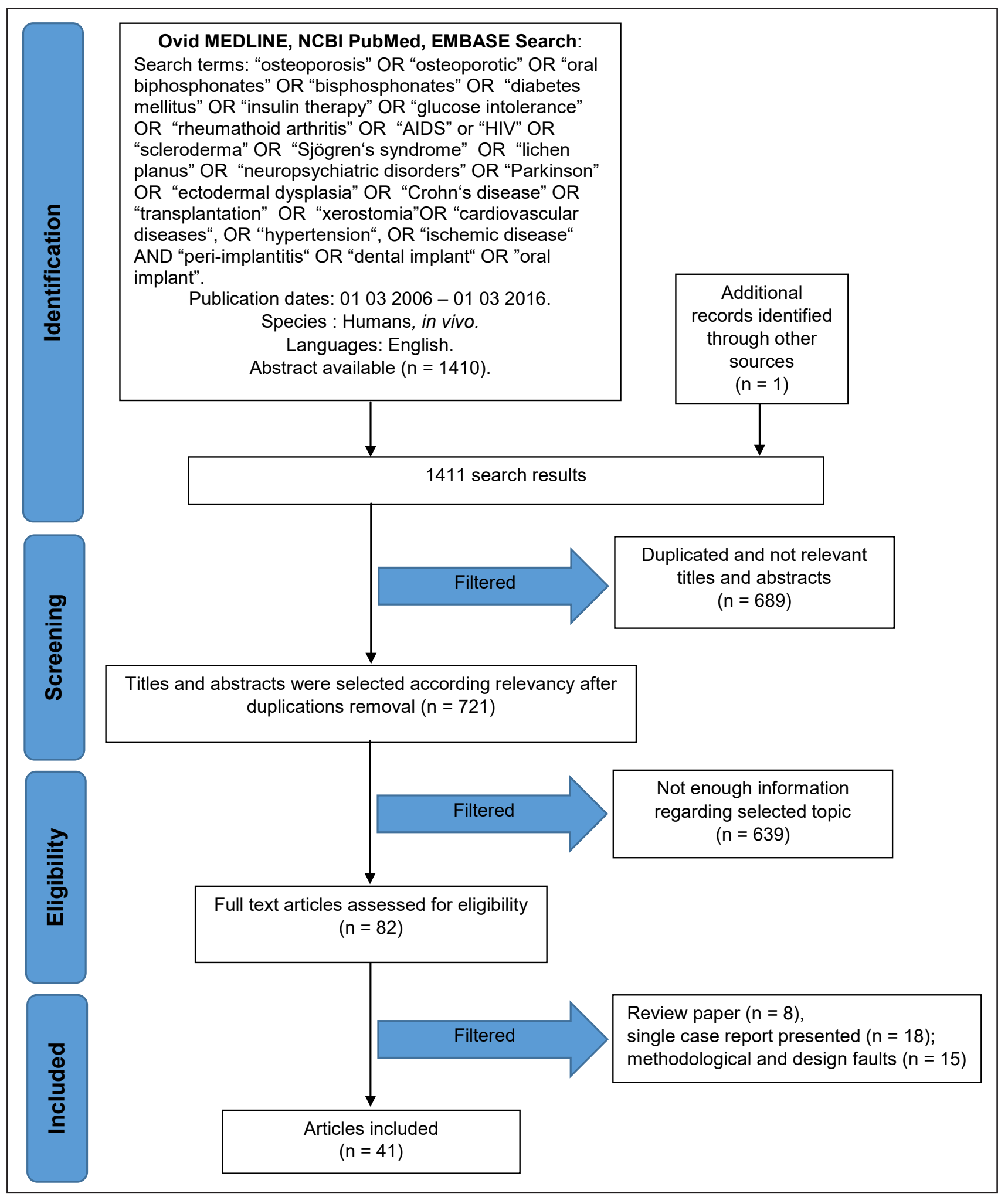

Figure 1. PRISMA flow diagram.

All studies were classified according to the disease as a risk factor to implant treatment type. 19 articles evaluated osteoporosis and/or bisphosphonate (BP) therapy effect [14-32], in six studies diabetes mellitus was analysed [33-38]. Five articles were based on cardiovascular influence evaluation [47,51-54], three authors reported the relation between implantation success and rheumatoid arthritis [41-43]. In two studies the effect of HIV was analysed $[\underline{39}, \underline{40}]$. One by one articles were included in evaluating the effect of following diseases and stages: Crohn's disease [47], Sjögren's syndrome [49], ectodermal dysplasia [50] and immunosuppression after transplantation [48]. 


\section{Risk of bias within the studies}

In total 13 studies were considered as "low risk of bias", since all the categories in Cochrane Collaboration's two-part tool were carried out with low possibility of bias [13]. Eleven reports were rated as "unclear risk of bias". 17 articles were decided to be "high risk of bias" due to methodological uncertainties. Most discrepancies were found in study sample selection and allocation processes (Table 1).

Table 1. Quality assessment

\begin{tabular}{|c|c|c|c|c|c|c|c|c|}
\hline Study & $\begin{array}{c}\text { Year of } \\
\text { publication }\end{array}$ & \begin{tabular}{|c|}
$\begin{array}{c}\text { Random } \\
\text { sequence } \\
\text { generation }\end{array}$ \\
\end{tabular} & $\begin{array}{c}\text { Allocation } \\
\text { concealment }\end{array}$ & \begin{tabular}{|c|} 
Blinding of \\
participants \\
and personnel \\
\end{tabular} & $\begin{array}{c}\text { Blinding of } \\
\text { outcome } \\
\text { assessment }\end{array}$ & \begin{tabular}{|c|} 
Incomplete \\
outcome \\
data
\end{tabular} & \begin{tabular}{|c|} 
Selective \\
reporting
\end{tabular} & \begin{tabular}{|c} 
Other \\
sources of \\
bias
\end{tabular} \\
\hline Kuchler et al. [14] & 2011 & + & + & + & $?$ & + & + & + \\
\hline Mozzati et al. [15] & 2015 & - & + & + & + & + & + & + \\
\hline Holahan et al. [16] & 2009 & - & + & + & + & - & + & + \\
\hline Shibli et al. [17] & 2007 & - & $?$ & + & + & + & + & + \\
\hline Kwon et al. [18] & 2012 & - & - & $?$ & + & + & + & + \\
\hline Siebert et al. [19] & 2015 & $?$ & $?$ & + & + & $?$ & + & + \\
\hline Jacobsen et al. [20] & 2012 & + & + & + & + & + & + & + \\
\hline Shabestari et al. [21] & 2009 & $?$ & + & + & + & + & + & + \\
\hline Lopez-Cedrum et al. [22] & 2013 & + & + & + & + & + & $?$ & + \\
\hline Goss et al. [23] & 2010 & - & - & + & + & $?$ & + & + \\
\hline Koka et al. [24] & 2010 & + & + & + & + & - & + & + \\
\hline Zahid et al. [25] & 2011 & + & + & + & + & + & + & + \\
\hline Grant et al. [26] & 2008 & + & + & + & + & - & + & + \\
\hline Lazarovici et al. [27] & 2010 & + & + & + & + & + & + & + \\
\hline Griffiths et al. [28] & 2012 & + & + & + & + & + & + & + \\
\hline Martin et al. [29] & 2010 & + & $?$ & + & + & $?$ & + & + \\
\hline Bell et al. [30] & 2010 & + & + & + & + & + & + & + \\
\hline Fugazzotto et al. [31] & 2007 & + & + & + & + & + & + & + \\
\hline Alsaadi et al. [32] & 2007 & $?$ & + & + & + & $?$ & + & + \\
\hline Turkyilmaz et al. [33] & 2010 & - & - & + & + & + & + & + \\
\hline Anner et al. [34] & 2010 & + & + & + & + & $?$ & $?$ & + \\
\hline Oates et al. [35] & 2009 & - & $?$ & + & + & $?$ & + & + \\
\hline Dowell et al. [36] & 2007 & - & + & + & + & + & + & + \\
\hline Tawil et al. [37] & 2008 & - & - & + & + & + & + & + \\
\hline Khandelwal et al. [38] & 2011 & + & + & + & + & + & + & + \\
\hline Oliveira et al. [39] & 2011 & - & - & + & + & + & + & + \\
\hline Stevenson et al. [40] & 2007 & - & $?$ & + & + & + & + & + \\
\hline Krennmair et al. [41] & 2010 & + & + & + & + & + & + & + \\
\hline Welnalder et al. [42] & 2010 & + & + & + & + & + & + & + \\
\hline Carr et al. [43] & 2010 & $?$ & $?$ & + & + & - & + & + \\
\hline Hernandez et al. [44] & 2012 & - & - & + & + & + & + & + \\
\hline Lopez-Jornet et al. [45] & 2014 & + & + & + & + & + & + & + \\
\hline Czerninski et al. [46] & 2013 & + & $?$ & + & + & $?$ & + & + \\
\hline Alsaadi et al. [47] & 2008 & $?$ & $?$ & + & + & + & + & + \\
\hline Montebugnoli et al. [48] & 2012 & - & - & + & + & + & + & + \\
\hline Korfage et al. [49] & 2015 & + & + & + & + & + & + & + \\
\hline Guckes et al. [50] & 2002 & $?$ & $?$ & + & + & $?$ & + & + \\
\hline Alsaadi et al. [51] & 2008 & + & + & + & + & $?$ & + & + \\
\hline Souza et al. [52] & 2013 & + & + & + & + & + & + & + \\
\hline Krennmair et al. [53] & 2016 & - & $?$ & + & + & + & + & + \\
\hline Renvert et al. [54] & 2013 & + & + & + & + & + & + & + \\
\hline
\end{tabular}

$+=$ low risk of bias; $?=$ unclear risk of bias; $-=$ high risk of bias. 


\section{Risk of bias across the studies}

Some of the limitations may be found in this review that may influence the outcomes. In present review only studies published in English were evaluated. Also, studies with small sample sizes were excluded, same as case reports. Also, high heterogeneity across the studies was seen. While most of the studies were retrospective, evaluating the broad period results, even prospective studies showed great methodological differences. Some studies measured the implantation results in patients with systemic diseases with results, provided from healthy participants, while other studies presented only systemic disease affected patients or those with complications. While some rare but severe complications were measured across the studies (e.g. Bisphosphonate related osteonecrosis of the jaws [BRONJ]) we have missed concrete risk rating in most of the studies. Also, in many studies various implantation manipulations were included with summed up results.

\section{Effect of various diseases on implantation outcomes Osteoporosis and bisphosphonates treatment}

Out of 4 studies, evaluating only osteoporosis as a risk factor to implantation success, none of them showed significant differences (Table 2) [14-17].

Kuchler et al. [14] study noted that the sample size was not enough to find any statistically significant differences to evaluate the effect of Teriparatide. Three other studies [15-17] did not find any significant differences for implantation success neither for osseointegration quality between osteoporotic and control groups. Holahan et al. [16] found that osteoporosis did not show any correlation to local jaw bone quality, but when it was, set subjectively by surgeon, it highly correlated with implantation success $(\mathrm{P}<0.01)$. It should be noted that Mozzati et al. $[15]$ combined many different implantation methods, such as immediate function loading, immediate implantation and sinus lifting. Sinus lift was mentioned as relevant implant failure risk factor. Also, patients with various risk factors, including diabetes mellitus, smoking, corticosteroid users, oral BP users, were combined in this study [15].

Totally different from all others in methodology, was Shibli et al. [17] study, in which histological evaluation was held. There were no statistically significant differences found between osteoporotic and control patients groups, according to the bone to implant contact (46 [SD 11.46]\% vs. 47.84 [SD $14.03] \%$ respectively) [17]. However, Alsaadi et al. [32] reported significant correlation between the osteoporosis and implant failure $(\mathrm{P}=0.001)$.
BRONJ as a severe complication, which could be provoked because of the implantation, was examined in various studies (Table 3) $[\underline{15}, \underline{18}, \underline{19}, \underline{22}, \underline{26}, \underline{27}, \underline{31]}$. Two main methodological groups could be concluded - studies $[\underline{15}, \underline{19}, \underline{26}, \underline{31}]$, evaluating the BRONJ among implantation patients, and studies $[\underline{18}, \underline{20}, \underline{22}, \underline{27}]$, evaluating the effect of implantation between BRONJ patients, presenting different outcomes.

Results from studies evaluating the percentage of BRONJ between osteoporotic, BP using implantation patients did not show any significant correlations $[\underline{15}, \underline{19}, \underline{26}, \underline{31}]$.

Authors that evaluated the implantation influence in BP users among the patients with BRONJ showed conflicting results. Four authors $[\underline{18}, \underline{20}, \underline{22}, \underline{23}]$ stated that implantation may induce the start of BRONJ in BP patients. However, Lazarovici et al. [27] stated, that surgical procedure is not a trigger for the BRONJ onset, since most BRONJ are late complications after implantation. All authors $[18,20,22,23,27]$ stated that BRONJ could be present even if the BP treatment is started after the full implants osseointegration and usually comes as late complication, which onsets after 20.9 months [20] to 35 months [18].

BP effect to the implantation success did not show any statistically significant differences in various studies $[\underline{21}, \underline{24}, \underline{25}, \underline{30}, \underline{31}]$. However, Zahid et al. [25] showed high correlation between the BP usage and implant thread exposure $(\mathrm{P}=0.001)$ among the 51 implants. Martin et al. [29] and Alsaadi et al. [32] found, that oral BP may cause various peri-implant complications $(P=0.001)[\underline{32}]$

Griffiths et al. [28] pilot study showed the effect of Aledronate to bone mineral density. Despite the small sample size $(n=10)$, placebo-drug trial revealed that Aledronate interferes bone remodelling in drug using period. Decreasing bone mineral density was present in results, during the alendronate intake, but 6 months after the end of drugs usage the bone mineral density rebounded. Nevertheless this was only a pilot study [28]; the BP effect to the bone - stronger or weaker is clearly present.

\section{Diabetes mellitus}

Half of the authors $[\underline{33}, \underline{34}, \underline{36}]$ did not find any significant correlations between the presence of diabetes mellitus and implantation success (Table 4). On the contrary, Dowell et al. [36] did not find any evidence of lower implantation success in poorly controlled patients. In one study [38] exclusively only poorly controlled diabetes mellitus patients were included a despite some complications (soft tissues inflammation, implant rotation, pain during 
Table 2. The basic information from articles, evaluating osteoporosis influence on implantation success

\begin{tabular}{|c|c|c|c|c|c|c|c|c|c|c|}
\hline \multirow[b]{2}{*}{ Study } & \multirow[b]{2}{*}{ Study type } & \multirow{2}{*}{$\begin{array}{c}\text { Follow-up } \\
\text { (period or mean) }\end{array}$} & \multirow[b]{2}{*}{ Sample size } & \multicolumn{2}{|c|}{ Number of implants } & \multicolumn{2}{|c|}{ Early complications } & \multicolumn{3}{|c|}{ Late complications } \\
\hline & & & & Maxilla & Mandible & $\begin{array}{l}\text { Patients } \\
(\%)\end{array}$ & $\begin{array}{c}\text { Implants } \\
(\%)\end{array}$ & $\begin{array}{l}\text { Years after } \\
\text { restauration }\end{array}$ & $\begin{array}{l}\text { Patients } \\
(\%)\end{array}$ & $\begin{array}{c}\text { Implants } \\
(\%)\end{array}$ \\
\hline Kuchler et al. [14] & Prospective & 9 weeks & 24 & 0 & 48 & 0 & 0 & 0 & 0 & 0 \\
\hline Mozzati et al. [15] & Retrospective & $2-9$ years & 235 & 660 & 607 & Unknown & Unknown & 5 & 6.8 & 1.3 \\
\hline \multirow{4}{*}{ Holahan et al. [16] } & \multirow{4}{*}{ Retrospective } & \multirow{4}{*}{6,1 years } & \multirow{4}{*}{$\begin{array}{c}645 \text { (all) } \\
208 \text { (with BMD-T scores within } 3 \text { years) }\end{array}$} & \multirow{5}{*}{\multicolumn{2}{|c|}{$\begin{array}{l}2867 \text { (all) } \\
701 \text { (with BMD-T scores within } 3 \text { years) }\end{array}$}} & \multirow{5}{*}{ Unknown } & \multirow{4}{*}{ Unknown } & 1 & & $\begin{array}{l}\text { Bone quality: } \\
\text { good 2.7; moderate } 4.6 \text {; poor } 11.6 \text {. }\end{array}$ \\
\hline & & & & & & & & 3 & & $\begin{array}{l}\text { Bone quality: } \\
\text { good 4.2; moderate } 7.1 \text {; poor } 16.6 \text {. }\end{array}$ \\
\hline & & & & & & & & 5 & & $\begin{array}{l}\text { Bone quality: } \\
\text { good } 5 ; \text { moderate } 7.5 \text {; poor } 17.4 \text {. }\end{array}$ \\
\hline & & & & & & & & 10 & & $\begin{array}{l}\text { Bone quality: } \\
\text { good } 6.7 ; \text { moderate } 8.8 \text {; poor } 17.4 \text {. }\end{array}$ \\
\hline Shibli et al. [17] & Retrospective & 4 years & 21 & & & & - & - & - & \\
\hline
\end{tabular}

BMD- $\mathrm{T}=$ Bone mineral density test.

Table 3. The basic information from articles, evaluating bisphosphonates influence on implantation success

\begin{tabular}{|c|c|c|c|c|c|c|c|c|c|c|c|c|}
\hline \multirow[b]{2}{*}{ Study } & \multirow[b]{2}{*}{ Study type } & \multirow[b]{2}{*}{ Follow-up } & \multirow[b]{2}{*}{ Sample size } & \multicolumn{2}{|c|}{ Number of implants } & \multicolumn{2}{|c|}{ Early complications } & \multicolumn{3}{|c|}{$\begin{array}{l}\text { Late complications } \\
\end{array}$} & \multicolumn{2}{|c|}{ All complications } \\
\hline & & & & Maxilla & Mandible & $\begin{array}{c}\begin{array}{c}\text { Patients } \\
(\%)\end{array} \\
\end{array}$ & $\begin{array}{c}\text { Implants } \\
(\%)\end{array}$ & $\begin{array}{c}\text { Years after } \\
\text { restauration }\end{array}$ & $\begin{array}{c}\text { Patients } \\
(\%)\end{array}$ & $\begin{array}{c}\text { Implants } \\
(\%)\end{array}$ & $\begin{array}{c}\text { Patients } \\
(\%)\end{array}$ & $\begin{array}{c}\text { Implants } \\
(\%)\end{array}$ \\
\hline Kwon et al. [18] & Prospective & $>3$ months & 19 & \multicolumn{2}{|c|}{ Unknown } & & & - & & & & \\
\hline Siebert et al. [19] & Prospective & 1 year & 24 (12 control, 12 BP+osteop) & & 120 & 0 & 0 & - & 0 & 0 & 0 & 0 \\
\hline Jacobsen et al. [20] & Retrospective & 5 years & 12 & 5 & 18 & - & - & - & & & & \\
\hline Shabestari et al. [21] & Case series & $<8$ year & 21 & 20 & 26 & 0 & 0 & - & 0 & 0 & 0 & 0 \\
\hline Lopez-Cedrum et al. [22] & $\begin{array}{l}\text { Retrospective. } \\
\text { Case series }\end{array}$ & $<3$ year & 9 & 28 & 29 & - & - & - & - & - & - & - \\
\hline Goss et al. [23] & Retrospective & Unknown & 7 & \multicolumn{2}{|c|}{19 (non-distinguished) } & - & - & - & - & - & - & - \\
\hline Koka et al. [24] & Retrospective & Unknown & 137 (55 BP and 82 non-BP) & \multicolumn{2}{|c|}{121 (BP) and 166 (non-BP) } & Unknown & Unknown & - & Unknown & Unknown & Unknown & $\begin{array}{l}1.81(\mathrm{BP}-) \\
0.83(\mathrm{BP}+)\end{array}$ \\
\hline Zahid et al. [25] & Retrospective & 26 months & 300 (274BP- and 26 BP+) & \multicolumn{2}{|c|}{661 (610 BP- and 51 BP+) } & $11.54 \%(\mathrm{BP}+)$ & $5.89 \%(\mathrm{BP}+)$ & - & Unknown & Unknown & $11.54 \%(\mathrm{BP}+)$ & $\begin{array}{r}2.9 \% \text { (all) } \\
5.89 \%(\mathrm{BP}+)\end{array}$ \\
\hline Grant et al. [26] & Retrospective & Unknown & 458 (343 non $\mathrm{BP}$ and $115 \mathrm{BP})$ & \multicolumn{2}{|c|}{1450 (non BP) 468 (BP) } & $1.7 \%(2 / 115)$ & $0.4 \%(2 / 468)$ & - & Unknown & Unknown & Unknown & $0.4 \%(\mathrm{BP}+)$ \\
\hline Lazarovici et al. [27] & Retrospective & 11.4 & 145 & Unknown & Unknown & - & - & - & - & - & - & \\
\hline Griffiths et al. [28] & Prospective & 18 months & 10 & \multicolumn{2}{|c|}{14 (non-distinguished) } & 0 & 0 & - & 0 & 0 & 0 & 0 \\
\hline Martin et al. [29] & Retrospective & Unknown & 589 (16 failed implants) & \multirow{2}{*}{\multicolumn{2}{|c|}{44 implants in 16 patients ( 26 failed) }} & & - & - & & & & \\
\hline Bell et al. [30] & Retrospective & Unknown & 42 & & & $11.9 \%$ & $5 \%$ & - & Unknown & Unknown & $11.9 \%$ & $5 \%$ \\
\hline Fugazzotto et al. [31] & Retrospective & $12-24$ months & 61 & \multicolumn{2}{|c|}{$\begin{array}{l}100 \text { (non-distinguished) } \\
169 \text { (non-distinguished) }\end{array}$} & 0 & 0 & - & 0 & 0 & 0 & 0 \\
\hline Alsaadi et al. [32] & Retrospective & Unknown & 2004 & \multicolumn{2}{|c|}{6946 (non-distinguished) } & $8.9 \%$ & $3.6 \%$ & & 0 & 0 & $8.9 \%$ & $3.6 \%$ \\
\hline
\end{tabular}

$\mathrm{BP}=$ bisphosphonate.

Table 4. The basic information from articles, evaluating diabetes mellitus influence on implantation success

\begin{tabular}{|c|c|c|c|c|c|c|c|c|c|c|}
\hline \multirow[b]{2}{*}{ Study } & \multirow[b]{2}{*}{ Study type } & \multirow[b]{2}{*}{$\begin{array}{c}\text { Follow-up } \\
\text { (mean) }\end{array}$} & \multirow[b]{2}{*}{ Sample size } & \multicolumn{2}{|c|}{ Number of implants } & \multicolumn{2}{|r|}{ Early complications } & \multicolumn{3}{|c|}{ Late complications } \\
\hline & & & & Maxilla & Mandible & $\begin{array}{l}\text { Patients } \\
\text { (\%) }\end{array}$ & $\begin{array}{c}\text { Implants } \\
(\%)\end{array}$ & $\begin{array}{l}\text { Period after } \\
\text { restauration }\end{array}$ & $\begin{array}{l}\text { Patients } \\
\text { (\%) }\end{array}$ & $\begin{array}{c}\text { Implants } \\
\text { (\%) }\end{array}$ \\
\hline Turkyilmaz et al. [33] & Retrospective & 1 years & 10 & 12 & 11 & 0 & 0 & - & 0 & 0 \\
\hline Anner et al. [34] & Retrospective & 30.82 months & 475 (49 DM+; 426 DM-) & 1626 (non & istinguished) & Unknown & Unknown & 24.71 months & $\begin{array}{c}12.2 \\
8.2(\mathrm{CD}+) \\
12.7(\mathrm{CD}-) \\
\end{array}$ & $\begin{aligned} 4,7 \\
2.8(\mathrm{CD}+) \\
5(\mathrm{CD}-) \\
\end{aligned}$ \\
\hline Oates et al. [35] & Prospective Pilot & 4 months & 32 & 42 (non-c & tinguished) & 0 & 0 & - & 0 & 0 \\
\hline Dowell et al. [36] & Prospective Pilot & 4 months & 35 & 16 & 34 & 0 & 0 & - & 0 & 0 \\
\hline Tawil et al. [37] & Prospective & 42.4 months & 90 (45 DM+; 45 DM-) & $499(255 \mathrm{D}$ & $1+; 244$ DM-) & Unknown & $\begin{array}{c}0.97 \% \text { (in }<7 \% \mathrm{HbAlc} \mathrm{gr} \text { ) } \\
3.5 \% \text { (in } 7-9 \% \mathrm{HbAlc} \text { gr) } \\
9 \% \text { (in a group of one person with }>9 \% \mathrm{HbAlc} \text { ) }\end{array}$ & 3 years & $2(1$ patient $\mathrm{DM}+)$ & 0.4 (1 implant) \\
\hline Khandelwal et al. [38] & $\begin{array}{l}\text { Prospective } \\
\text { (controlled trial) }\end{array}$ & 16 weeks & 24 & & 8 & Unknown & $\begin{array}{c}38 \% \text { (SLA) } \\
33 \% \text { (modSLA) } \\
\text { Not osseointegrated: } 2 \% \text { (1 SLA implant) } \\
\end{array}$ & - & 0 & 0 \\
\hline
\end{tabular}

$\mathrm{DM}^{+}=$diabetes mellitus patients; $\mathrm{DM}-=$ diabetes mellitus free; $\mathrm{HbA1C}=$ glycated haemoglobin; $\mathrm{SLA} / \mathrm{modSLA}=$ specific implant type, reported by author 
the healing abutment application) were observed, at all $98 \%$ successes was reached.

Oates et al. [35] found that osseointegration during the first 4 months postoperatively was poor in people with higher HbAlc levels. Only 57.1\% implants of poorly controlled diabetes mellitus returned to exceeded baseline stability level after 16 week, compared with $80 \%$ in well-controlled group [35]. The similar outcomes were reached in Tawil et al. [37] study, where statistically significant correlations between HbA1c levels and peri-implantitis $(\mathrm{P}=0.05)$ or periimplant bone loss $(\mathrm{P}=0.01)$ were observed.

The higher risk of implantation in diabetes mellitus patients was mentioned in all the studies, despite the positive outcomes [33-38].

\section{HIV and AIDS}

Two studies $[39,40]$ were identified as representatives for implant therapy for HIV patients. Evidence level is limited due to low number of patients and short observation period. However, there were no differences in implant success among the groups with HIV- or HIV+ $[\underline{39}, \underline{40}]$.

\section{Rheumatoid arthritis}

Three studies were enrolled to show the influence of rheumatoid arthritis on implant treatment success [41-43]. The success rates range between 93.8\% [41] and 96,1\% [42]. None of the authors found significant correlations between the rheumatoid arthritis and implantation success [41-43]. However, one author [41] stated that the crestal bone resorption and bleeding on probing was higher in patients with rheumatoid arthritis.

\section{Oral lichen planus}

Three studies with the different study type and follow-up periods were analysed to evaluate influence of oral lichen planus (OLP) on the of periimplant complications [44-46]. Authors of all studies concluded that success of implant rehabilitation among treated OLP patients does not differ from the success rate in the general population. Implant survival and success rate was $100 \%$ vs. $96.8 \%$ in the control group [44]. Two studies confirmed that implant placement didn't influence OLP manifestation $[\underline{45}, \underline{46}]$.

\section{Crohn's disease}

Only one prospective study included in the review shows statistically significant

Crohn's disease's effect on early implant failures [47]. However evidence level is limited as this study included 11 patients with the 6 months follow-up.

\section{Immunosuppression after organ transplantation}

One prospective controlled study was included that shows effect of immunosuppressive therapy on the patient's that undergo organ transplantation [48]. Despite small sample size of 10 patients and 20 implants with the follow-up three months, Montebugnoli et al. [48] concluded that the bone response around submerged dental implants in immunocompromised organ transplant patients does not differ from that observed in control patients and no rejection of implants was noticed. There is no data on the success of implants in the long term.

\section{Sjögren's syndrome}

One retrospective study was identified that shows success of implantation in 50 patient's group with Sjögren's syndrome [49]. Early complications rate was $3 \%$ of 140 implants. Although patients showed more signs of peri-implant mucositis, Korfage et al. [49] stated this clinically irrelevant, and after median follow-up of 3.8 years prevalence of peri-implantitis was $11 \%$ which is comparable with healthy patients. There is some caution with regard to the potentially increased risk on peri-implantitis in the long term [49].

\section{Ectodermal dysplasia}

Only one study included involving patients with ectodermal dysplasia [50]. The short-term survival data supports successful placing of dental implants in patients with ectodermal dysplasia and congenitally missing teeth with appropriate precautions in the maxilla. Although the age of these patients ranged from 8 to 68 years, there was no significant difference in survival of implants among patient age groups. The difference in the survival rates of implants placed in anterior mandible (9\%) and maxilla (23\%) approached significance [무].

\section{Cardiovascular diseases}

All five studies included to assess impact of cardiovascular diseases (CVD) on implantation outcomes are dealing with several correlative factors (other systemic diseases, smoking, periodontitis) and CVD are among them [느, 51-54]. 
Alsaadi et al. [51] in short term study concluded that tendency for early failures wasn't noticed for CVD or hypertension.

de Souza et al. [52] investigated 189 patients with CVD and hypertension out of 253 individuals that had previously attended the supportive periodontal program, to assess the impact of local and systemic factors on additional bone loss (ABL $>2 \mathrm{~mm}$ according to the X-ray). Analysis included former chronic periodontitis diagnosis; smoking and local factors and confirmed that CVD are not associated with additional bone loss [51]. Alsaadi et al. [47] in retrospective study found that late implant loss was not correlating with CVD and hypertension.

On the contrary, results of two studies out of five state that patients with CVD showed increased peri-implant bone loss [53] and periimplantitis is associated with CVD [54].

Krennmair et al. [53] revealed that patients with CVD $(n=19)$ show significantly increased periimplant marginal bone loss for 4 implant supported mandibular full arch restorations. However study investigated small sample of cardiac patients including smokers and assessed marginal bone loss only, there is no data published on the success rate of implants [53].

A history of CVD was found in $27.3 \%$ if individuals with peri-implantitis and in $3 \%$ of individuals in the implant health/peri-implant mucositis group [54]. The present study assessed data on radiographic bone levels and concluded that peri-implantitis is a high likelihood of comorbidity expressed by a history of CVD and periodontitis [54].

\section{DISCUSSION}

In present review we have established only few systemic diseases that were approved by several authors to significantly affect implant treatment success.

Most studies were based on evaluating the relationship between the osteoporosis and its treatment influence on implantation success. Osteoporosis is a systemic bone disorder, which is related with the loss of internal structure loss in bone's architecture, lower bone mass and micro and macro bone changes $[\underline{55}, \underline{56}]$. There are several different osteoporosis aetiology theories, asserting the lack of oestrogen, calcium circulation disorders, thyroid dysfunction as main causing factor $[\underline{55}, \underline{57}, \underline{58}]$. Due to the demonstrated effect of oestrogen insufficiency, in many studies only post-menopausal females were included $[\underline{15}, \underline{16}, \underline{19}, \underline{24}, \underline{26}]$. However, in none of the studies laboratorial hormonal examinations were performed, only stating that post-menopausal women are in higher risk for osteoporosis without individual examinations. Despite the pathogenic osteoporosis effect to the bone, Holahan et al. [16] did not find any significant relationships between the osteoporosis and local bone mineral density in jaws. Most authors stated that there aren't any statistically significant correlations between the osteoporosis and implantation success [14-17]. However, it should be mentioned, that BP, the mostly used in osteoporosis treatment, may show some different results. The main working mechanism is based on suppression of both: bone remodelling and osteoclastic activity, and support of stable structure [59]. However, rare, but severe side effects are known due to use of BP, such as osteonecrosis of jaws, which usually arise in cancer patients with high BP doses undergoing invasive dental procedures [ 55,59]. Also, bone healing is usually prolonged in BP treated patients because of bone remodelling suppression [55]. While in present review half of the authors stated, that the connection between BRONJ and implantation is not statistically significant $[15,19, \underline{26}, \underline{31}]$, the other half of the articles $[18,20,22,23,27]$ showed that BRONJ may appear not only as implantation triggered procedure, but even after the full implants osseointegration, when treatment with BP starts. These differences, in present authors' opinion, appeared due to the methodological and included subjects differences. While the BRONJ among all implantation patients, undertaking BP, is rare, from those with BRONJ, the implantation as possible causative factor is more likely to be. In five studies any negative BP effect to implantation was declined $[\underline{21}, \underline{24}, \underline{25}, \underline{30}, \underline{31}]$, but two authors stated, that the risk of peri-implant lesions is higher in patients undertaking BP [29,32]. Even if aledronates and risedronates are the first line medicine used in osteoporosis treatment [ $\underline{59}$ ], aledronate was found to be the most correlated BP with implant failures [22].

Most authors did not find the negative diabetes mellitus effect to the implantation outcomes $[\underline{33}, 34,36]$, even in poorly controlled diabetes mellitus patients [38]. The main measurement tool used was HbA1c plasma levels. However, World Health Organization states that $\mathrm{HbAlc}$ monitoring is not a suitable test to measure intermediate hyperglycaemia [60]. HbA1c shows mean plasma glucose levels of past several weeks, so this method should be used to assess the control of present diabetes mellitus, not the disease itself $[\underline{56}, \underline{61}]$. Nevertheless, Tawil et al. [구] found statistically significant relationship between the plasma $\mathrm{HbA} 1 \mathrm{c}$ levels and peri-implant lesions. Also, prolonged healing was found in non-well-controlled 
diabetes mellitus patients [35]. This is not surprising, since generally wound healing is prolonged in diabetes mellitus patients due to the lack of growth factors, impaired macrophage function, collagen accumulation, keratinocytes and fibroblasts migration processes [62]. Also, angiogenesis is poor in diabetes mellitus patients, which could be critical factor in voluminous surgical procedures [62].

Rheumatoid arthritis is complex systemic disease and the main mechanism is associated with autoimmune reactions that cause a destruction of articulatory systems. [63,64] Multiple cytokines play important role in promoting synovial inflammations, that later proceed to destructive process. [63-65] Bone destruction process is caused because of high osteoclastic activity, induced by inflammatory mediators and macrophages that are present in rheumatoid arthritis synovitis [63] However, despite these mechanisms, none of the authors found correlations with implantation treatment failures [41-43]. Even if implantations were successful in all studies, Krennmair et al. [41] stated that crestal bone resorption and bleeding after the probing was higher in rheumatoid arthritis patients. This might be due to the low disease expression to the implantation sites, or due to the methodological causes. To resolve this question, further studies are needed, since pathogenesis shows possible severe effect.

OLP is one of the most common chronic inflammatory autoimmune diseases that involve the oral mucosa with an incidence of $1-2 \%$ in various populations [66]. Several studies selectively exclude implantation in subjects with OLP $[\underline{67}, \underline{68}]$. Possible risks of dental implant placement in erosive OLP were suggested by Lekholm [69] who hypothesised an increased risk of failure due to the altered capability of the epithelium to adhere to the implant surface.

However according to this review success of implant rehabilitation among treated OLP patients does not differ from the success rate in the general population [44-46] and implant placement didn't influence OLP manifestation $[\underline{45}, \underline{46}]$.

This opinion is confirmed by other systematic reviews $[70,71]$. In a case of OLP implants should be positioned only if mucosal signs and symptoms are in the remission phase. A careful oral hygiene and frequent follow-up are the main recommendations in OLP patients rehabilitated with implants $[\underline{69}, \underline{71}]$. With caution due to the level of scientific evidence of the analysed studies, OLP does not influence the success of dental implant therapy.

Evidence for implantation outcomes in patients with Crohn's disease, Sjögren's syndrome, ectodermal dysplasia and immunosuppressive state after transplantation due to the single publications, small sample sizes, short term follow-up is very low.

Crohn's disease as an autoimmune disease, affects host response, gastro-intestinal system and leads to periodontal lesions, osseointegration failures [72]. According to Alsaadi et al. [47] Crohn's disease may negatively influence osseointegration success, still further studies are needed to provide these findings.

Immunosuppressive treatment that follows organ transplantation may have negative effect on the bone mineral metabolism [73]. However single study of Montebugnoli et al. [48] indicated absence of early complications and showed that the bone response around submerged dental implants in immunocompromised organ transplant patients does not differ from that observed in control patients. The same was found with human immunodeficiency virus evaluation, since both studies evaluating HIV effect on implantation success did not show any evidence of negative effect to the procedure outcomes $[39,40]$

Outcome of dental implants in patients with Sjögren's syndrome and ectodermal dysplasia seem comparable to those of healthy patients $[\underline{49}, \underline{50}]$, but further clinical investigations including control groups with healthy patients with longer follow-up are needed to clear provided information.

CVD may compromise blood flow and reduce oxygen and nutrient supply to tissues. This may have influence the outcome of osseointegration and induce crestal bone level changes over the time [53].

Implantation success data for cardiovascular patients are controversial and should be evaluated with caution. Results of two studies out of five $[47, \underline{51-}$ 54] state that patients with CVD showed increased peri-implant bone loss [53] and periimplantitis is associated with CVD [54].

All the studies are dealing with several factors, among these is cardiovascular. Regression analysis might be an answer in a population with multiple diseases, study by Alsaadi et al. [51] states that systemic health factors do not seem to be prominent players in the aetiology of late implant loss. However, Mombelli and Cionca [8] in the review study, considered the fact that potential risk factors, particularly those found more frequently in older adults in general systemic chronic diseases, smoking, periodontitis history, medications, reduced salivary flow - may not be independent of each other. One single factor alone may not influence the risk measurably, while a combination of multiple, independent factors may have a significant impact [ $\underline{8}]$.

Other limitations of these studies which might explain their controversy, are heterogeneous data structure, some of them are unavailable as cardiovascular 
patients consisted only part of whole sample; no data published on the success rate of implants [47,53]; implants with different surfaces included [54]. Radiographic examination of additional bone loss has limitation due to the fact that it is performed only in two dimensions, which is not possible to examine buccal and oral sites [52].

Influence of systemic health problems to periimplantitis requires additional epidemiologic and clinical investigations. For higher state of evidence randomized controlled clinical trials with included at least 10 patients per condition, more than 1 year of follow-up after implant loading, and the homogeneous criteria (probing pocket depth, bleeding on probing and/or pus, marginal bone loss) to define periimplantitis.

\section{Limitations}

In present review we have faced some limitations, due to the high risk of bias assessed in various articles. Most included studies were retrospective, which could not give the full information about the implantation procedure implementation and possible risk of bias, reached before the evaluation process. Also, because those only English articles were included, some data could be missing.

\section{CONCLUSIONS}

1. Osteoporosis is not an absolute contraindication to implantation.

2. Implantation in bisphosphonate users may result in rare but severe osteonecrosis complication, regardless of the time of bisphosphonate treatment start - prior or post implantation.

3. Diabetes mellitus is still controversially assessed in different studies; however it is clearly characterized as a factor encumbering implantation post-operative healing process.

4. Oral lichen planus and rheumatoid arthritis was rated only in few studies, nevertheless no effect of these diseases to implantation success was found.
5. Evidence level of implantation success in HIV/ AIDS is limited due to few studies with short follow-up and small sample sizes, in which no effect to implantation was found.

6. Influence of cardiovascular diseases on dental implantation success should be explored deeply, since controversial results were assessed. For higher state of evidence detailed randomized controlled clinical trials with homogeneous criteria are needed.

7. To clarify the effect of Crohn's disease to implantation success, more studies are needed, since only one prospective study with unclear risk of bias and small sample size was found with no evidence of Crohn's disease negative influence on implantation outcomes.

8. Sjögren's syndrome, immunosuppressive state after transplantation and ectodermal dysplasia are rather non-related with implantation therapy success, but due to the single publications, small sample sizes, short term follow-up and possible risk of bias these evidences for implantation outcomes are very low.

9. Clear and homogenous methodology is needed since heterogeneous studies outcomes comparison is complicated and not highly reliable.

10. In studies, including patients with multiple diseases, possible co-effect of various diseases should also be reflected before excluding single disorders.

11. There were no studies evaluating xerostomia and neuropsychiatric disorders, such as Parkinson's disease, impact on implantation success that met present review inclusion criteria.

\section{ACKNOWLEDGMENTS AND DISCLOSURE STATEMENTS}

Authors would like to give acknowledgments to all the people that help in assessing the articles in interest, without any authorship.

No funding was needed to proceed with present review.

\section{REFERENCES}

1. Gómez-de Diego R, Mang-de la Rosa Mdel R, Romero-Pérez MJ, Cutando-Soriano A, López-ValverdeCenteno A. Indications and contraindications of dental implants in medically compromised patients: update. Med Oral Patol Oral Cir Bucal. 2014 Sep 1;19(5):e483-9. [Medline: 24608222] [PMC free article: 4192572] [doi: $10.4317 /$ medoral.19565]

2. Turri A, Rossetti PH, Canullo L, Grusovin MG, Dahlin C. Prevalence of Peri-implantitis in Medically Compromised Patients and Smokers: A Systematic Review. Int J Oral Maxillofac Implants. 2016 Jan-Feb;31(1):111-8. [Medline: 26800167] [doi: 10.11607/jomi.4149] 
3. Serino G, Ström C. Peri-implantitis in partially edentulous patients: association with inadequate plaque control. Clin Oral Implants Res. 2009 Feb;20(2):169-74. [Medline: 19077152] [doi: 10.1111/j.1600-0501.2008.01627.x]

4. Marrone A, Lasserre J, Bercy P, Brecx MC. Prevalence and risk factors for peri-implant disease in Belgian adults. Clin Oral Implants Res. 2013 Aug;24(8):934-40. [Medline: 22551347] [doi: 10.1111/j.1600-0501.2012.02476.x]

5. Sgolastra F, Petrucci A, Severino M, Gatto R, Monaco A. Periodontitis, implant loss and peri-implantitis. A meta-analysis. Clin Oral Implants Re. 2015 Apr;26(4):e8-16. [Medline: 24382358] [doi: 10.1111/clr.12319]

6. Smolik I, Robinson D, El-Gabalawy HS. Periodontitis and rheumatoid arthritis: epidemiologic, clinical, and immunologic associations. Compend Contin Educ Den. 2009 May;30(4):188-90. [Medline: 19441735]

7. Sonnenschein SK, Meyle J. Local inflammatory reactions in patients with diabetes and periodontitis. Periodontol 2000. 2015 Oct;69(1):221-54. [Medline: 26252411] [doi: 10.1111/prd.12089]

8. Mombelli A, Cionca N. Systemic diseases affecting osseointegration therapy. Clin Oral Implants Res. 2006 Oct;17 Suppl 2:97-103. [Medline: 16968386] [doi: 10.1111/j.1600-0501.2006.01354.x]

9. Diz P, Scully C, Sanz M. Dental implants in the medically compromised patient. J Dent. 2013 Mar;41(3):195-206. Epub 2013 Jan 11. [Medline: 23313715] [doi: 10.1016/j.jdent.2012.12.008]

10. Buser D, von Arx T, ten Bruggenkate C, Weingart D. Basic surgical principles with ITI implants. Clin Oral Implants Res. 2000;11 Suppl 1:59-68. [Medline: 11168257] [doi: 10.1034/j.1600-0501.2000.011S1059.x]

11. Moher D, Liberati A, Tetzlaff J, Altman DG, The PRISMA Group. Preferred Reporting Items for Systematic Reviews and Meta-Analyses: The PRISMA Statement. J Clin Epidemiol 2009: 62:1006-1012. [Medline: 19631508] [doi: 10.1016/j.jclinepi.2009.06.005]

12. Viera AJ, Garrett JM. Understanding interobserver agreement: the kappa statistic. Fam Med. 2005 May;37(5):360-3. [Medline: 15883903]

13. Higgins JPT, Green S. Cochrane Handbook for Systematic Reviews of Interventions version 5.1.0. The Cochrane Collaboration, 2011. [URL: http://training.cochrane.org/handbook]

14. Kuchler U, Luvizuto ER, Tangl S, Watzek G, Gruber R. Short-term teriparatide delivery and osseointegration: a clinical feasibility study. J Dent Res. 2011 Aug; 90(8):1001-6. [Medline: 21555773] [doi: 10.1177/0022034511407920]

15. Mozzati M, Arata V, Giacomello M, Del Fabbro M, Gallesio G, Mortellaro C, Bergamasco L. Failure risk estimate after dental implants placement associated with plasma rich grow factor-Endoret in osteoporotic women under bisphosphonate therapy. J Craniofac Surg. 2015 May;26(3):749-55. [Medline: 25974785] [doi: 10.1097/SCS.0000000000001535]

16. Holahan CM, Wiens JL, Weaver A, Assad D, Koka S. Relationship between systemic bone mineral density and local bone quality as effectors of dental iplant survival. Clin Implant Dent Relat Res. 2011 Mar;13(1):29-33. [Medline: 19681944] [doi: 10.1111/j.1708-8208.2009.00206.x]

17. Shibli JA, Aguiar KC, Melo L, d'Avila S, Zenobio EG, Faveri M, Iezzi G, Piattelli A. Histological comparison between implants retrieved from patients with and without osteoporosis. Int J Oral Maxillofac Surg. 2008 Apr;37(4):321-7. [Medline: 18262765] [doi: 10.1016/j.ijom.2007.11.019]

18. Kwon TG, Lee CO, Park JW, Choi SY, Rijal G, Shin HI. Osteonecrosis associated with dental implants in patients undergoing bisphosphonate treatment. Clin Oral Implants Res. 2014 May;25(5):632-40. [Medline: 23278625] [doi: 10.1111/clr.12088]

19. Siebert T, Jurkovic R, Statelova D, Strecha J. Immediate Implant Placement in a Patient With Osteoporosis Undergoing Bisphosphonate Therapy: 1-Year Preliminary Prospective Study. J Oral Implantol. 2015 Jul;41 Spec No:360-5. [Medline: 23278625] [doi: 10.1563/AAID-JOI-D-13-00063]

20. Jacobsen C, Metzler P, Rösle M, Obwegeser J, Zemann W, Gratz KW. Osteopathology induced by bisphosphonates and dental implants: clinical observations. Clin Oral Investig. 2013 Jan;17(1):167-75. [Medline: 22415216] [doi: 10.1007/ s00784-012-0708-2]

21. Shabestari GO, Shayesteh YS, Khojasteh A, Alikhasi M, Moslemi N, Aminian A, Masaeli R, Eslami B, Treister NS. Implant placement in patients with oral bisphosphonate therapy: a case series. Clin Implant Dent Relat Res. 2010 Sep;12(3):175-80. [Medline: 19438964]

22. López-Cedrún JL, Sanromán JF, García A, Penarrocha M, Feijoo JF, Limeres J, Diz P. Oral bisphosphonate-related osteonecrosis of the jaws in dental implant patients: a case series. Br J Oral Maxillofac Surg. 2013 Dec;51(8):874-9. [Medline: 23866309] [doi: 10.1016/j.bjoms.2013.06.011]

23. Goss A, Bartold M, Sambrook P, Hawker P. The nature and frequency of bisphosphonate-associated osteonecrosis of the jaws in dental implant patients: a South Australian case series. J Oral Maxillofac Surg. 2010 Feb;68(2):337-43. [Medline: 20116705] [doi: 10.1016/j.joms.2009.09.037]

24. Koka S, Babu NM, Norell A. Survival of dental implants in post-menopausal bisphosphonates users. J Prosthodont Res. 2010 Jul;54(3):108-11. [Medline: 20434422] [doi: 10.1016/j.jpor.2010.04.002]

25. Zahid TM, Wang BY, Cohen RE. Influence of bisphosphonates on alveolar bone loss around osseointegrated implants. J Oral Implantol. 2011 Jun;37(3):335-46. [Medline: 20594057] [doi: 10.1563/AAID-JOI-D-09-00114]

26. Grant BT, Amenedo C, Freeman K, Kraut RA. Outcomes of placing dental implants in patients taking oral bisphosphonates: a review of 115 cases. J Oral Maxillofac Surg. 2008 Feb;66(2):223-30. [Medline: 18201600] [doi: 10.1016/j.joms.2007.09.019] 
27. Lazarovici TS, Yahalom R, Taicher S, Schwatrz-Arad D, Peleg O, Yarom N. Bisphosphonate-related osteonecrosis of the jaw associated with dental implants. Oral Maxillofac Surg. 2010 Apr;68(4):790-6. [Medline: 20307764] [doi: 10.1016/j.joms.2009.09.017]

28. Griffiths GR. Bone density around endosseous implants in patients taking alendronate: a pilot study. Int J Periodontics Restorative Dent. 2012 Jun;32(3):e101-8. [Medline: 22408779]

29. Martin DC, O’Ryan FS, Indresano AT, Bogdanos P, Wang B, Hui RL, Lo JC. Characteristics of implant failures in patients with a history of oral bisphosphonate therapy. J Oral Maxillofac Surg. 2010 Mar;68(3):508-14. [Medline: 20171469] [doi: 10.1016/j.joms.2009.09.055]

30. Bell BM, Bell RE. Oral bisphosphonates and dental implants: a retrospective study. Oral Maxillofac Surg. 2008 May; 66(5):1022-4. [Medline: 18423296] [doi: 10.1016/j.joms.2007.12.040]

31. Fugazzotto PA, Lighfoot WS, Jaffin R, Kumar A. Implant placement with or without simultaneus tooth extraction in patients taking oral bisphosphonates: postoperative healing, early follow-up, and the incidence of complications in two private practices. J Periodontol. 2007 Sep;78(9):1664-9. [Medline: 17760533] [doi: 10.1902/jop.2007.060514]

32. Alsaadi G, Quirynen M, Komarek A, van Steenberghe D. Impact of local and systemic factors on the incidence of oral implant failures, up to abutment connection. J Cil Periodontol. 2007 Jul;34(7):610-7. [Medline: 17433044] [doi: 10.1111/j.1600-051X.2007.01077.x]

33. Turkyilmaz I. One-year clinical outcome of dental implants placed in patients with type 2 diabetes mellitus: a case series. Implant Dent. 2010 Aug;19(4):323-9. [Medline: 20683289] [doi: 10.1097/ID.0b013e3181e40366]

34. Anner R, Grossmann Y, Anner Y, Levin L. Smoking, diabetes mellitus, periodontitis, and supportive periodontal treatment as factors associated with dental implant survival: a long-term retrospective evaluation of patients followed for up to 10 years. Implant Dent. 2010 Feb;19(1):57-64. [Medline: 20147817] [doi: 10.1097/ID.0b013e3181bb8f6c]

35. Oates TW, Dowell S, Robinson M, McMahan CA. Glycemic control and implant stabilization in type 2 diabetes mellitus. J Dent Res. 2009 Apr;88(4):367-71. [Medline: 19407159] [PMC free article: 2904396] [doi: $10.1177 / 0022034509334203$ ]

36. Dowell S, Oates TW, Robinson M. Implant success in people with type 2 diabetes mellitus with varying glycemic control: a pilot study. J Am Dent Assoc. 2007 Mar;138(3):355-61; quiz 397-8. [Medline: 17332041] [doi: 10.14219/jada.archive.2007.0168]

37. Tawil G, Younan R, Azar P, Sleilati G. Conventional and advanced implant treatment in the type II diabetic patient: surgical protocol and long-termclinical results. Int J Oral Maxillofac Implants. 2008 Jul-Aug;23(4):744-52. [Medline: 18807573]

38. Khandelwal N, Oates TW, Vargas A, Alexander PP, Schoolfield JD, Alex McMahan C. Conventional SLA and chemically modified SLA implants in patients with poorly contolled type 2 diabetes mellitus - a randomized controlled trial. Clin Oral Implants Res. 2013 Jan;24(1):13-9. [Medline: 22145978] [doi: 10.1111/j.1600-0501.2011.02369.x]

39. Oliveira MA, Gallottini M, Pallos D, Maluf PS, Jablonka F, Ortega KL. The success of endosseous implants in human immunodeficiency virus-positive patients receiving antiretroviral therapy: a pilot study. J Am Dent Assoc. 2011 Sep;142(9):1010-6. [Medline: 21881066] [doi: 10.14219/jada.archive.2011.0320]

40. Stevenson GC, Riano PC, Moretti AJ, Nichols CM, Engelmeier RL, Flaitz CM. Short-term success of osseointegrated dental implants in HIV-positive individuals: a prospective study. J Contemp Dent Pract. 2007 Jan 1;8(1):1-10. [Medline: 17211499 ]

41. Krennmair G, Seemann R, Piehslinger E. Dental implants in patients with rheumatoid arthritis: clinical outcome and peri-implant findings. J Clin Periodontol. 2010 Oct;37(10):928-36. [Medline: 20718894] [doi: 10.1111/j.1600-051X.2010.01606.x]

42. Weinlander M, Krennmair G, Piehslinger E. Implant prosthodontic rehabilitation of patients with rheumatic disorders: a case series report. Int J Prosthodont. 2010 Jan-Feb;23(1):22-8. [Medline: 20234887]

43. Carr AB. Implant location and radiotherapy are the only factors linked to 2-year implant failure. J Evid Based Dent Pract. 2012 Sep;12(3 Suppl):217-9. [Medline: 23253850] [doi: 10.1016/S1532-3382(12)70042-8]

44. Hernandez G, Lopez-Pintor Rm, Arriba L, Torres J, de Vicente JC. Implant treatment in patients with oral lichen planus: a prospective-controlled study. Clin Oral Implants Res. 2012 Jun; 23(6):726-32. [Medline: 21492237] [doi: 10.1111/j.1600-0501.2011.02192.x]

45. López-Jornet P, Camacho-Alonso F, Sánchez-Siles M. Dental implants in patients with oral lichen planus: a cross-sectional study. Clin Implant Dent Relat Res. 2014 Feb;16(1):107-15. [Medline: 24533567] [doi: 10.1111/j.1708-8208.2012.00455.x]

46. Czerninski R, Eliezer M, Wilensky A, Soskolne A. Oral lichen planus and dental implants--a retrospective study. Clin Implant Dent Relat Res. 2013 Apr;15(2):234-42. [Medline: 21599828] [doi: 10.1111/j.1708-8208.2011.00347.x]

47. Alsaadi G, Quirynen M, Michiles K, Teughels W, Komarek A, van Steenberghe D. Impact of local and systemic factors on the incidence of failures up to abutment connection with modified surface oral implants. J Clin Periodontol. 2008 Jan;35(1):51-7. [Medline: 18034851]

48. Montebugnoli L, Venturi M, Cervellati F. Bone response to submerged implants in organ transplant patients: a prospective controlled study. Int J Oral Maxillofac Implants. 2012 Nov-Dec;27(6):1494-500. [Medline: 23189301] 
49. Korfage A, Raghoebar GM, Arends S, Meiners PM, Visser A, Kroese FG, Bootsma H, Vissink A. Dental Implants in patients with Sjögren's Syndrome. Clin Implant Dent Relat Res. 2015 Sep 24. [Medline: 26399938]

50. Guckes AD, Scurria MS, King TS, McCarthy GR, Brahim JS. Prospective clinical trial of dental implants in persons with ectodermal dysplasia. J Prosthet Dent. 2002 Jul;88(1):21-5. [Medline: 12239475] [doi: 10.1067/mpr.2002.127099]

51. Alsaadi G, Quirynen M, Komárek A. Impact of local and systemic factors on the incidence of late oral implant loss. Clin Oral Implants Res. 2008 Jul;19(7):670-6. [Medline: 18492080]

52. de Souza JG, Neto AR, Filho GS, Dalago HR, de Souza Junior JM, Bianchini MA. Impact of local and systemic factors on additional peri-implant bone loss. Quintessence Int. 2013 May;44(5):415-24. [Medline: 23479580]

53. Krennmair S, Weinländer M, Forstner T. Factors affecting peri-implant bone resorption in 4 implant supported mandibular full-arch restorations: a 3-year prospective study. J Clin Periodontol. 2016 Jan;43(1):92-101. [Medline: 26445457] [doi: $10.1111 /$ jcpe.12469]

54. Renvert S, Aghazadeh A, Hallström H, Persson GR. Factors related to peri-implantitis - a retrospective study. Clin Oral Implants Res. 2014 Apr;25(4):522-9. [Medline: 23772670] [doi: 10.1111/clr.12208]

55. Chew SL, Leslie D. Clinical endocrinology and diabetes: an illsutrated colour text. UK, Churchill Livingstone; 2006. p. 55.

56. Urbanavicius V. [Cukrinio diabeto klinika, diagnostika ir gydymas]. Vilnius: Vaistų žinios; 2009. Lithuanian.

57. Raisz LG. Pathogenesis of osteoporosis: concepts, conflicts, and prospects. J Clin Invest. 2005 Dec;115(12):3318-25. Review. [Medline: 16322775] [PMC free article: 297264] [doi: 10.1172/JCI27071]

58. Bono CM, Einhorn TA. Overview of osteoporosis: pathophysiology and determinants of bone strength. Eur Spine J. 2003 Oct;12 Suppl 2:S90-6. Epub 2003 Sep 12. Review. [Medline: 13680312] [PMC free article: 297264] [doi: 10.1007/s00586-003-0603-2]

59. Sandhu SK, Hampson G. The pathogenesis, diagnosis, investigation and management of osteoporosis. J Clin Pathol. 2011 Dec;64(12):1042-50. Epub 2011 Sep 6. Review. [Medline: 21896577] [doi: 10.1136/jep.2010.077842]

60. World Health organization. Definition and diagnosis of diabetes mellitus and intermediate hyperglycemia. Geneva: World Health Organization; 2006. p. 1-46. Report of a WHO/IDF Consultation. [URL: http://www.who.int/diabetes/publications/diagnosis_diabetes2006/en/]

61. Sultanpur CM, Deepa K, Kumar SV. Comprehensive review on hbalc in diagnosis of diabetes mellitus. Int J Pharm Sci Rev Res. 2010 Aug; 3(2): 119-22. [URL: http://www.globalresearchonline.net/pharmajournal/pharmajournal6.aspx]

62. Brem H, Tomic-Canic M. Cellular and molecular basis of wound healing in diabetes. J Clin Invest. 2007 May;117(5):121922. Review. [Medline: 17476353] [PMC free article: 1857239] [doi: 10.1172/JCI32169]

63. Choy E. Understanding the dynamics: pathways involved in the pathogenesis of rheumatoid arthritis. Rheumatology (Oxford). 2012 Jul;51 Suppl 5:v3-11. [Medline: 22718924]

64. Firestein GS. Etiology and pathogenesis of rheumatoid arthritis. In: Harris ED, Budd RC, Genovese MC, Firestein GS, Sargent JS, Sledge CB, editors. Kelley‘s Textbook of Rheumatology. 7th ed. Philadelphia, Pa: Saunders Elsevier; 2005. p. 1059-108.

65. Aletaha D, Neogi T, Silman AJ, Funovits J, Felson DT, Bingham CO 3rd, Birnbaum NS, Burmester GR, Bykerk VP, Cohen MD, Combe B, Costenbader KH, Dougados M, Emery P, Ferraccioli G, Hazes JM, Hobbs K, Huizinga TW, Kavanaugh A, Kay J, Kvien TK, Laing T, Mease P, Ménard HA, Moreland LW, Naden RL, Pincus T, Smolen JS, StanislawskaBiernat E, Symmons D, Tak PP, Upchurch KS, Vencovsky J, Wolfe F, Hawker G. 2010 rheumatoid arthritis classification criteria: an American College of Rheumatology/European League Against Rheumatism collaborative initiative. Ann Rheum Dis. 2010 Sep;69(9):1580-8. Erratum in: Ann Rheum Dis. 2010 Oct;69(10):1892. [Medline: 20699241] [doi: 10.1136/ard.2010.138461]

66. Carrozzo M, Thorpe R. Oral lichen planus: a review. Minerva Stomatol. 2009 Oct;58(10):519-37. Review. English, Italian. [Medline: 19893476 ]

67. Thomsson M, Esposito M. A retrospective case series evaluating Branemark BioHelix implants placed in a specialist private practicefollowing 'conventional' procedures. One-year results after placement. Eur J Oral Implantol. 2008 Autumn;1(3):229-34. [Medline: 20467625]

68. Chiapasco M, Abati S, Romeo E, Vogel G. Implant-retained mandibular overdentures with Brånemark System MKII implants: a prospective comparativestudy between delayed and immediate loading. Int J Oral Maxillofac Implants. 2001 Jul-Aug;16(4):537-46. [Medline: 11516001]

69. Lekholm U. The surgical site. In: Clinical periodontology and implant dentistry, 4th edn. Lindhe J, editor. Copenhagen: Blackwell Munksgaard; 2003. p. 854.

70. Petruzzi M, De Benedittis M, Cortelazzi R, Milillo L, Lucchese A, Serpico R, Grassi FR. Implant rehabilitation in patients with oral lichen planus: an overview. Clin Oral Investig. 2012 Oct;16(5):1347-52. [Medline: 22382447] [doi: 10.1007/s00784-012-0695-3]

71. Reichart PA, Schmidt-Westhausen AM, Khongkhunthian P, Strietzel FP. Dental implants in patients with oral mucosal diseases - a systematic review. J Oral Rehabil. 2016 May;43(5):388-99. [Medline: 26685871] [doi: 10.1111/joor.12373]

72. van Steenberghe D, Jacobs R, Desnyder M, Maffei G, Quirynen M. The relative impact of local and endogenous patient-related factors on implant failure up to the abutment stage. Clin Oral Implants Res. 2002 Dec;13(6):617-22. [Medline: 12519336] [doi: 10.1034/j.1600-0501.2002.130607.x] 
73. Stein E, Ebeling P, Shane E. Post-transplantation osteoporosis.Endocrinol Metab Clin North Am. 2007 Dec;36(4): 937-63; viii. [Medline: 17983930] [doi: 10.1016/j.ecl.2007.07.008]

\section{To cite this article:}

Guobis Z, Pacauskiene I, Astramskaite I.

General Diseases Influence on Peri-Implantitis Development: a Systematic Review

J Oral Maxillofac Res 2016;7(3):e5

URL: http://www.ejomr.org/JOMR/archives/2016/3/e5/v7n3e5.pdf

doi: $10.5037 /$ jomr.2016.7305

Copyright (c) Guobis Z, Pacauskiene I, Astramskaite I. Published in the JOURNAL OF ORAL \& MAXILLOFACIAL RESEARCH (http://www.ejomr.org), 9 September 2016.

This is an open-access article, first published in the JOURNAL OF ORAL \& MAXILLOFACIAL RESEARCH, distributed under the terms of the Creative Commons Attribution-Noncommercial-No Derivative Works 3.0 Unported License, which permits unrestricted non-commercial use, distribution, and reproduction in any medium, provided the original work and is properly cited. The copyright, license information and link to the original publication on (http://www.ejomr.org) must be included. 